\title{
Global Validity of the Boltzmann Equation for a Two-Dimensional Rare Gas in Vacuum
}

\author{
Reinhard Illner ${ }^{1}$ and Mario Pulvirenti ${ }^{2}$ \\ 1 Fachbereich Mathematik, Universität Kaiserslautern, Erwin-Schrödinger-Straße, D-6750 Kaisers- \\ lautern, Federal Republic of Germany \\ 2 Dipartimento di Matematica dell' Università “La Sapienza”, P.A. Moro, I-00185 Roma, Italy
}

\begin{abstract}
We consider a system of $N$ hard disks in $\mathbb{R}^{2}$ in the Boltzmann-Grad limit (i.e. $N \rightarrow \infty, d \searrow 0, N \cdot d \rightarrow \lambda^{-1}>0$, where $d$ is the diameter of the disks). If $\lambda$ is sufficiently small and if the joint distribution densities factorize at time zero, we prove that the time-evolved one-particle distribution converges for all times to the solution of the Boltzmann equation with the same initial datum.
\end{abstract}

\section{Introduction}

It is generally believed that in certain limit situations the dynamics of a gas of particles can be described by the Boltzmann equation. One of the basic problems in the foundations of kinetic theory is to prove the validity of this statement in a rigorous way, assuming, as a starting point, the laws of classical mechanics. The difficulty and appeal of this problem stem from the necessity that one has to relate two evolutions with very different natures: Newtonian dynamics, which are deterministic and reversible, and Boltzmann dynamics, which have a stochastic character and are irreversible.

A first result in this direction was obtained by Lanford [1] who deduced, in a rigorous way, the validity of the Boltzmann equation for short times (on the order of magnitude of a fraction of the mean free time).

In this paper we consider a two-dimensional system of hard disks and prove, by following the general strategy proposed in [1], the validity of the Boltzmann equation for all times in the case of a gas allowed to expand into free space and for large enough mean free paths (in comparison with the initial datum). In doing this we use the idea developed in [2] for solving the Cauchy problem for the Boltzmann equation in the corresponding three-dimensional situation-namely, that free flow has good contracting properties on functions rapidly decreasing at infinity.

We discuss the limitations of our result. The method applies to cases in which the density decays at infinity, so thermodynamical situations elude our analysis. Furthermore, we are not able to treat more realistic three-dimensional systems. This is probably a technical difficulty. Finally, we assume that the gas be rarefied in the 
sense that the mean free path has to be large enough. Although our estimates are not optimal, this constraint could be really essential, also from a physical point of view. In any case: In order to answer the validity question for the Boltzmann equation, one first has to solve the associated Cauchy problem, and a positive answer for this problem, globally-in-time, is not yet known in the general case.

\section{Formulation of the Problem and Main Results}

We consider the time evolution of $N$ identical disks in $\mathbb{R}^{2}$, interacting by means of elastic collisions. We denote by $\phi_{t}^{d} X=\left(x_{1}^{d}(t), \ldots, x_{N}^{d}(t), v_{1}^{d}(t), \ldots, v_{N}^{d}(t)\right)$ the positions and velocities of the particles at time $t$, where $X=\left(x_{1}, \ldots, x_{N}, v_{1}, \ldots, v_{N}\right)$ denotes the phase point at time 0 and $d$ is the diameter of the particles. The flow $\phi_{t}^{d} X$ is defined almost everywhere with respect to the Liouville measure $d X=d x_{1} \cdots d x_{N} d v_{1} \cdots d v_{N}$. Let

$$
\mu^{d}(d X)=\mu^{d}(X) d X
$$

be an absolutely continuous probability measure on the phase space of the system and

$$
f_{k}^{d}\left(x_{1}, \ldots, x_{k}, v_{1}, \ldots, v_{k}\right)=\int \mu^{d}(X) d x_{k+1} \cdots d x_{N} d v_{k+1} \cdots d v_{N}
$$

the joint distribution densities. We assume $\mu^{d}$ (and hence the $f_{k}^{d}$ ) to be symmetric with respect to the variables $q_{i}=\left(x_{i}, v_{i}\right)$, because the particles are indistinguishable.

Let

$$
\mu_{t}^{d}(X) d X=\mu^{d}\left(\phi_{-t}^{d} X\right) d X
$$

be the time evolved measure and let $f_{k, t}^{d}$ be the associated joint distribution densities. Then

$$
f_{k, t}^{d}=S^{d}(t) f_{k}^{d}+\int_{0}^{t} d s S^{d}(t-s) C_{k, k+1}^{d} f_{k+1, s}^{d}
$$

where

$$
\begin{gathered}
f_{j, t}^{d} \equiv 0 \quad \text { if } j>N, \\
\left(S^{d}(t) f_{k}^{d}\right)\left(x_{1} \cdots x_{k}, v_{1} \cdots v_{k}\right)=f_{k}^{d}\left(\phi_{-t}^{d}\left(x_{1} \cdots x_{k}, v_{1} \cdots v_{k}\right)\right), \\
C_{k, k+1}^{d} f_{k+1}^{d}\left(x_{1} \cdots x_{k}, v_{1} \cdots v_{k}\right) \\
=\sum_{j=1}^{k}(N-k) d \int d n \int d v_{k+1} n \cdot\left(v_{j}-v_{k+1}\right) f_{k+1}^{d}\left(x_{1} \cdots x_{k} x_{j}+d \cdot n, v_{1} \cdots v_{k} v_{k+1}\right),
\end{gathered}
$$

and $n$ denotes a unit vector in $\mathbb{R}^{2}$.

The derivation of the hierarchy of Eqs. (2.4) can, e.g., be found in [3], but the discussion there is largely formal. The hierarchy corresponds to the well-known BBGKY-hierarchy, usually established for particles interacting via smooth potentials, and is a consequence of the laws of classical mechanics. From a rigorous point of view the meaning of Eqs. (2.4) is not completely clear, because the flow $\phi_{t}^{d}$ is only almost everywhere defined. We face the problem of giving sense to the collision operator $C_{k, k+1}^{d}$ in Eq. (2.7) for $f_{k+1}^{d}$, which is only a.e. defined. However, we can give 
a meaning to the following series expansion associated with $(2.4)_{1}$ :

$$
S^{d}(-t) f_{k, t}^{d}=f_{k}^{d}+\sum_{n=1}^{N-k} \int_{0}^{t} d t_{1} \int_{0}^{t_{1}} d t_{2} \cdots \int_{0}^{t_{n}-1} d t_{n} S^{d}\left(-t_{1}\right) C_{k, k+1}^{d} S^{d}\left(t_{1}-t_{2}\right) \cdots S^{d}\left(t_{n}\right) f_{k+n}^{d}
$$

In fact, we denote by $\Gamma_{k}$ the $k$-particle phase space and choose $g_{k+1} \in L^{\infty}\left(\Gamma_{k+1}\right)$. Consider the configurations $X_{k} \in \Gamma_{k}$ and $y_{k+1} \in \mathscr{F}_{k+1}$, where $\mathscr{F}_{k+1}:=$ $\left\{X_{k+1} \in \Gamma_{k+1}|| x_{i}-x_{k+1} \mid=d\right.$ for some $\left.i\right\}$ denotes the set of all $k+1$-phase points obtained from an $X_{k}$ by adjoining a colliding particle, and study $\phi_{-t}^{d}\left(Y_{k+1}\right)$. If we vary $Y_{k+1}$ over $\mathscr{F}_{k+1}$ and $t \in[0, T], \phi_{-t}^{d}\left(Y_{k+1}\right)$ spans a tube flow which is a set of positive measure in $\Gamma_{k+1}$. Therefore $g_{k+1}\left(\phi_{-t}^{d}\left(Y_{k+1}\right)\right)$ is defined almost everywhere in $\mathscr{F}_{k+1} \times[0, T]$. Integrating with respect to $\sum_{i} d v_{k+1} d n n \cdot\left(v_{i}-v_{k+1}\right)$, we obtain a function, denoted by $C_{k, k+1} S^{d}(t) g_{k+1}$, which is defined a.e. in $\Gamma_{k} \times[0, T]$. The same argument shows that $S^{d}\left(-t_{1}\right) C_{k, k+1}^{d} S^{d}\left(t_{1}-t_{2}\right) \cdots S^{d}\left(t_{n}\right) f_{k+n}^{d}$ is defined for almost all $t_{n}<t_{n-1}<t_{n-2}<\cdots<t_{1}<t$ and almost all $x_{k}$. Thus the right-hand side of $(2.4)_{2}$ makes sense and "defines," for all $t$, a function $S^{d}(-t) f_{k, t}^{d}$ which is a.e. defined in $\Gamma_{k}$. From now on, $S^{d}(-t) f_{k, t}^{d}$ will always be interpreted as the right-hand side of $(2.4)_{2}$. The equivalence between the $S^{d}(-t) f_{k, t}^{d}$ defined by $(2.4)_{2}$ and the joint distribution densities given by (2.3) will be established in the appendix.

Besides Eqs. (2.4) we also consider the so-called Boltzmann hierarchy:

$$
f_{k, t}=S(t) f_{k}+\int_{0}^{t} d s S(t-s) C_{k, k+1} f_{k+1, s}
$$

where

$$
S(t) f_{k}\left(x_{1} \cdots x_{k}, v_{1} \cdots v_{k}\right)=f_{k}\left(x_{1}-v_{1} t \cdots x_{k}-v_{k} t, v_{1} \cdots v_{k}\right)
$$

describes free flow and

$$
\begin{aligned}
C_{k, k+1} f_{k+1}\left(x_{1} \cdots x_{k}, v_{1} \cdots v_{k}\right) \\
=\sum_{j=1}^{k} \lambda^{-1} \int d v_{k+1} \int_{n\left(v_{j}-v_{k+1}\right) \geq 0} d n n\left(v_{j}-v_{k+1}\right) \\
\quad \cdot\left\{f_{k+1}\left(x_{1} \cdots x_{k} x_{j}, v_{1} \cdots v_{j}^{\prime} \cdots v_{k} v_{k+1}^{\prime}\right)\right. \\
\left.\quad-f_{k+1}\left(x_{1} \cdots x_{k} x_{j}, v_{1} \cdots v_{j} \cdots v_{k} v_{k+1}\right)\right\} .
\end{aligned}
$$

Here, $v_{j}^{\prime}, v_{k+1}^{\prime}$ denote the post-collisional velocities belonging to the incoming velocities $v_{j}, v_{k+1}$ and the collision parameter $n$. $\lambda$ is a positive constant proportional to the mean free path between collisions.

If $f_{t}(x, v)$ is a solution of the Boltzmann equation for hard disks, then the functions

$$
f_{k, t}\left(x_{1} \cdots x_{k}, v_{1} \cdots v_{k}\right)=\prod_{j=1}^{k} f_{t}\left(x_{j}, v_{j}\right)
$$

satisfy the hierarchy (2.8). This explains the name of the hierarchy.

The similarity between Eqs. (2.4) and (2.8) becomes more transparent by 
rewriting the collision operator from Eqs. (2.7) in the equivalent form

$$
\begin{aligned}
& C_{k, k+1}^{d} f_{k+1, t}\left(x_{1} \cdots x_{k}, v_{1} \cdots v_{k}\right) \\
& =\sum_{j=1}^{k}(N-k) d \int d n \int_{n \cdot\left(v_{j}-v_{k+1}\right) \geqq 0} d v_{k+1} n\left(v_{j}-v_{k+1}\right) \\
& \quad \cdot\left\{f_{k+1, t}\left(x_{1} \cdots x_{j} \cdots x_{k} x_{j}-n d, v_{1} \cdots v_{j}^{\prime} \cdots v_{k+1}^{\prime}\right)\right. \\
& \left.\quad-f_{k+1, t}\left(x_{1} \cdots x_{j} \cdots x_{k} x_{j}+n d, v_{1} \cdots v_{j} \cdots v_{k+1}\right)\right\}
\end{aligned}
$$

(see [1] for details). In spite of the similarity, the hierarchies are very different. The first (finite) hierarchy corresponds to the time evolution of a reversible Hamiltonian system. The second one has a stochastic nature and displays irreversibility-we know that the $H$-theorem holds rigorously for short times. Nevertheless, Eqs. (2.12) are the formal limit as $d \rightarrow 0, N \rightarrow \infty, N \cdot d \rightarrow \lambda^{-1}$ (this is known as the BoltzmannGrad limit) of Eqs. (2.7), and therefore, in this limit situation, a particle gas is believed to behave according to (2.8).

We now formulate our result. Consider a sequence of statistical states $\left(\mu^{d}\right)$ associated with hard disk systems with $d>0$ and $N$ such that $N \rightarrow \infty, d \rightarrow 0$ and $\lambda^{-1}=N \cdot d$ fixed. We assume that the joint distribution densities $f_{j}^{d}$ at time zero satisfy

i) if $\left(\mathbb{R}^{2} \times \mathbb{R}^{2}\right)_{\neq}^{j, d}=\left\{X|| x_{i}-x_{k} \mid>d\right.$ for $\left.i \neq k, i, k=1, \ldots, j, d \geqq 0\right\}$, then the $f_{j}^{d}$ are continuous on $\left(\mathbb{R}^{2} \times \mathbb{R}^{2}\right)_{\neq}^{j, d}$. Furthermore, there exists an infinite sequence $\left\{f_{j}\right\}_{j}$, continuous on $\left(\mathbb{R}^{2} \times \mathbb{R}^{2}\right)_{\neq}^{j, 0}$, such that

$$
\lim _{d \rightarrow 0} f_{j}^{d}=f_{j}
$$

uniformly on compact subsets of $\left(\mathbb{R}^{2} \times \mathbb{R}^{2}\right)_{\neq \neq}^{j, 0}$.

ii) the estimates

$$
\sup _{\substack{x_{1} \ldots x_{j} \\ v_{1} \ldots v_{j}}}\left|f_{j}^{d}\left(x_{1} \cdots x_{j}, v_{1} \cdots v_{j}\right)\right| \exp \beta_{0}\left(\sum_{i=1}^{j}\left(x_{i}^{2}+v_{i}^{2}\right)\right) \leqq \text { const } z_{0}^{j}
$$

hold for some $\beta_{0} \geqq 2 \exp \left(\lambda^{-1}\right)$ and $z_{0}>0$.

Theorem 2.1. Assume i), ii) and let $z_{0} \cdot \lambda^{-1}$ be sufficiently small. Then, for all $t>0$ and $j>0$,

$$
\lim _{d \rightarrow 0} f_{j, t}^{d}\left(x_{1} \cdots x_{j}, v_{1} \cdots v_{j}\right)=f_{j, t}\left(x_{1} \cdots x_{j}, v_{1} \cdots v_{j}\right) \text { a.e. }
$$

where $f_{j, t}$ solves uniquely the Boltzmann hierarchy.

If the initial state factorizes, i.e. if

$$
f_{j}\left(x_{1} \cdots x_{j}, v_{1} \cdots v_{j}\right)=\prod_{k=1}^{j} f_{1}\left(x_{k}, v_{k}\right)
$$

then this factorization is preserved in time for $f_{j, t}$, and $f_{1, t}$ is a unique solution of the Cauchy problem for the Boltzmann equation.

Proof. Following [1] we write $(2.4)_{2}$,

$$
f_{t}^{d}=S^{d}(t) f^{d}+\sum_{n \geqq 1} \int_{0}^{t} d t_{1} \int_{0}^{t_{1}} d t_{2} \cdots \int_{0}^{t_{n}-1} d t_{n} S^{d}\left(t-t_{1}\right) C^{d} \cdots S^{d}\left(t_{n}\right) f^{d},
$$


where $f_{t}^{d}=\left\{f_{k, t}^{d}\right\}_{k=1}^{\infty}, S^{d}(t) f^{d}=\left\{S^{d}(t) f_{k}^{d}\right\}_{k=1}^{\infty}, C^{d} f=\left\{\left(C^{d} f\right)_{k}\right\}_{k=1}^{\infty}=\left\{C_{k, k+1}^{d} f_{k+1}\right\}_{k=1}^{\infty}$ (we recall that we are using the convention $f_{k, t}^{d}=0$ for $t \geqq 0$ and $k>N$, thus the series appearing on the right of (2.17) is only a finite sum). Analogously we write a similar (formal) expansion for the Boltzmann hierarchy, with initial datum given by the limit (2.13):

$$
f_{t}=S(t) f+\sum_{n \geqq 1} \int_{0}^{t} d t_{1} \int_{0}^{t_{1}} d t_{2} \cdots \int_{0}^{t_{n-1}} d t_{n} S\left(t-t_{1}\right) C \cdots S\left(t_{n}\right) f,
$$

with $S(t) f=\left\{S(t) f_{k}\right\}_{k=1}^{\infty}$ and $C f=\left\{(C f)_{k}\right\}_{k=1}^{\infty}=\left\{C_{k, k+1} f_{k+1}\right\}_{k=1}^{\infty}$. In [1] it is shown that under the hypotheses of Theorem 2 each term of the series (2.17) converges a.e. to the corresponding term of the perturbation series (2.18). Therefore, to prove (2.15) it is enough to show convergence of the Boltzmann hierarchy series and to bound the series (2.17) by a convergent series of nonnegative terms not depending on $d$.

To approach the last problem, we rewrite the series under consideration in a slightly different way:

$$
\begin{aligned}
& S^{d}(-t) f_{t}^{d}=f^{d}+\sum_{n \geqq 1} \int_{0}^{t} d t_{1} \int_{0}^{t_{1}} d t_{2} \cdots \int_{0}^{t_{n-1}} d t_{n} \\
& \Omega^{d}\left(t_{1}\right) V^{d}\left(t_{1}\right) \Omega^{d}\left(t_{1}\right)^{-1} \cdots \Omega^{d}\left(t_{n}\right) V^{d}\left(t_{n}\right) \Omega^{d}\left(t_{n}\right)^{-1} f^{d},
\end{aligned}
$$

where

and

$$
\Omega^{d}(t)=S^{d}(-t) S(t)
$$

$$
V^{d}(t)=S(-t) C^{d} S(t)
$$

For convenience, we assume that the $f_{j}^{d}$ are defined on all of $\left(\mathbb{R}^{2} \times \mathbb{R}^{2}\right)^{j}$, but are zero on sets which lead to the overlapping of spheres. Moreover, we define $\Omega^{d}(t)^{-1}$ to be the identity operator whenever $\phi_{t}(X)$ is not in the domain of definition of $\phi_{-t}^{d}$. This is reasonable because in this case $\Omega^{d}(t)^{-1}$ is not defined by

$$
\left(\Omega^{d}(t)\right)^{-1} f(X)=f\left(\phi_{-t}^{d}\left(\phi_{t}(X)\right)\right)
$$

The proof of Theorem 2.1 is based on the key Proposition 2.1 below which gives estimates on the operators $\Omega^{d}(t) V^{d}(t) \Omega^{d}(t)^{-1}$. We now introduce the spaces needed for the formulation of this result.

For $\beta>0$, let

$$
\left\|f_{j}\right\|_{\beta, j}=\underset{\substack{x_{1} \ldots x_{j} \\ v_{1} \ldots v_{j}}}{\operatorname{ess} \sup }\left|f_{j}\left(x_{1} \cdots x_{j}, v_{1} \cdots v_{j}\right)\right| \exp \beta \sum_{i=1}^{j}\left(x_{i}^{2}+v_{i}^{2}\right)
$$

and for $z>0$ we define a norm on the space of all sequences of measurable functions $f=\left\{f_{j}\right\}_{j=1,2 \ldots}$ by

$$
\|f\|_{\beta}^{z}=\sup _{j}\left\|f_{j}\right\|_{\beta, j} \cdot z^{-j}
$$

We denote by $X_{\beta, j}$ and $X_{\beta}^{z}$ the Banach spaces induced by the norms (2.22) and (2.23). Then we have

Proposition 2.1. Let $\tau>0$ be arbitrary but fixed and let $z<z^{\prime},(1+d) \quad \beta^{\prime}<\beta$, 
$d<1 / \sqrt{2} \tau^{2}$, and $g \in X_{\beta}^{z}$. Then there exists a positive constant $C_{0}>0$ (not depending on $\left.\tau, d, \beta, \beta^{\prime}, z, z^{\prime}\right)$ such that for almost all $t \in[0, \tau]$,

$$
\begin{gathered}
\left\|\Omega^{d}(t) V^{d}(t) \Omega^{d}(t)^{-1} g\right\|_{\beta^{\prime}}^{z^{\prime}} \leqq C_{0} z \lambda^{-1}\left(\frac{1}{1+t^{3}}\right) l(\beta) e^{2 \sqrt{ } 2 \beta} . \\
\cdot\left(\frac{1}{\left|\ln \frac{z^{\prime}}{z}\right|}+\frac{1}{\sqrt{\left|\ln \frac{z^{\prime}}{z}\right|\left(\beta-\beta^{\prime}(1+d)\right)}}\right)\|g\|_{\beta}^{z},
\end{gathered}
$$

where

$$
l(\beta)=\max \left(\beta^{-3 / 2}, \beta^{-1}\right) .
$$

For the proof see the next section.

By Proposition 2.1, we have for suitable sequences, $z=z_{n}>z_{n-1}>\cdots>z_{0}$, $\beta=\beta_{n}<\beta_{n-1}<\cdots<\widetilde{\beta}_{0}$, such that $\beta \geqq 1$ and $(1+d) \beta_{j}<\beta_{j-1}$, and for $t_{j}<\tau$ arbitrary but fixed, and $d<1 / \sqrt{2} \tau^{2}$, that

$$
\begin{aligned}
&\left\|\Omega^{d}\left(t_{1}\right) V^{d}\left(t_{1}\right) \Omega^{d}\left(t_{1}\right)^{-1} \ldots \Omega^{d}\left(t_{n}\right) V^{d}\left(t_{n}\right) \Omega^{d}\left(t_{n}\right)^{-1} f^{d}\right\|_{\beta}^{z} \\
& \leqq\left\|f^{d}\right\|_{\beta_{0}^{0}}^{z_{0}^{\circ}}\left(C_{0} \lambda^{-1}\right)^{n} \cdot \prod_{j=1}^{n}\left(\frac{1}{\ln \left|\frac{z_{j}}{z_{j-1}}\right|}+\frac{1}{\sqrt{\left|\ln \frac{z_{j}}{z_{j-1}}\right|\left(\beta_{j-1}-\beta_{j}(1+d)\right)}}\right) \\
& \cdot\left(\frac{z_{j-1} l\left(\beta_{j-1}\right) \exp \left(2 \sqrt{2} \beta_{j}\right)}{1+t_{j}^{3}}\right) .
\end{aligned}
$$

To estimate this, we choose the $z_{j}$ and $\beta_{j}$ such that

$$
\ln \frac{z_{j}}{z_{j-1}}=\frac{1}{n}\left(\text { i.e. } z_{j}=z_{j-1} e^{1 / n}=z_{0} e^{j / n}\right)
$$

and $\beta_{j-1}-\beta_{j}(1+d)=1 / n$ (and hence $\left.\widetilde{\beta}_{0}=1 / n+(1+d) / n+\cdots+(1+d)^{n} \beta_{n}\right)$. We conclude that $\tilde{\beta}_{0} \leqq 2 \cdot(1+d)^{n} \beta \leqq 2\left(1+\lambda^{-1} / n\right)^{n} \beta \leqq 2 \beta \cdot e^{\lambda^{-1}}$, because $n \leqq N=\lambda^{-1} / d$. (Note that here the dimension enters in a crucial way: In three dimensions the Boltzmann-Grad limit is $N \cdot d^{2}=\lambda^{-1}$, which only leads to the (insufficient) estimate $(1+d)^{n} \leqq\left(1+\sqrt{\lambda^{-1} / n}\right)^{n}$.) Let $\beta_{0}:=2 \beta \cdot e^{\lambda^{-1}}$. Inserting all this in the above expression, it follows that there is a constant $A_{0}>0$ such that,

$$
\begin{aligned}
& \left\|\Omega^{d}\left(t_{1}\right) V^{d}\left(t_{1}\right) \cdots V^{d}\left(t_{n}\right) \Omega^{d}\left(t_{n}\right)^{-1} f^{d}\right\|_{\beta}^{z} \\
& \quad \leqq\left\|f^{d}\right\|_{\beta_{0}}^{z_{0}}\left(A_{0} n \lambda^{-1} l(\beta)\left[\exp \left(\beta_{0} 2 \sqrt{2}\right)\right] z_{0}\right)^{n} \prod_{j=1}^{n}\left(\frac{1}{1+t_{j}^{3}}\right) .
\end{aligned}
$$

Therefore, if $f^{d} \in X_{\beta_{0}}^{z_{0}}$, we obtain after reordering and integration from zero to infinity

$$
\begin{aligned}
\left\|f_{j, t}^{d}\right\|_{\infty} & =\left\|S^{d}(-t) f_{j, t}^{d}\right\|_{\infty} \leqq z^{j}\left\|S^{d}(-t) f_{j, t}^{d}\right\|_{\beta}^{z} \\
& \leqq z^{j}\left(\left\|f_{j}^{d}\right\|_{\beta}^{z}+\sum_{n \geqq 1}^{t} \int_{0}^{t} d t_{1} \cdots \int_{0}^{t_{n}-1} d t_{n}\left\|\Omega^{d}\left(t_{1}\right) V^{d}\left(t_{1}\right) \Omega^{d}\left(t_{1}\right)^{-1} \cdots f^{d}\right\|_{\beta}^{z}\right) \\
& \leqq e^{j} z_{0}^{j} \sum_{n \geqq 0}\left(\int_{0}^{\infty} \frac{d s}{1+s^{3}}\right)^{n}\left(A_{0} \lambda^{-1} z_{0} l\left(\beta_{0} e^{-\lambda^{-1}}\right) e^{\left.2 \sqrt{ } 2 \beta_{0}\right)^{n}} \frac{n^{n}}{n !}\left\|f^{d}\right\|_{\beta_{0}}^{z_{0}} .\right.
\end{aligned}
$$


If $z_{0} \cdot \lambda^{-1}$ is sufficiently small, we see that the series (2.19) is estimated by a convergent series not depending on $d$.

For the Boltzmann hierarchy, let $V(t)=S(-t) C S(t)$. Then, following the proof of Proposition 3.2 in Sect. 3, one obtains an estimate corresponding to (2.24):

$$
\|V(t) f\|_{\beta^{\prime}}^{z^{\prime} \leqq z \cdot C l(\beta) \lambda^{-1}}\left(\frac{1}{1+t^{3}}\right)\left\{\frac{1}{\left|\ln \frac{z}{z^{\prime}}\right|}+\frac{1}{\sqrt{\left(\beta-\beta^{\prime}\right)\left|\ln \frac{z}{z^{\prime}}\right|}}\right\}\|f\|_{\beta}^{z},
$$

where $\beta>\beta^{\prime} \geqq 1, z^{\prime}>z>0$. Here, $C$ can be chosen independently of $z, z^{\prime}, \beta, \beta^{\prime}$, and the estimate holds for all $t>0$. Using this result, we can mimick the above convergence proof and obtain convergence for the Boltzmann hierarchy. The limit (2.15) follows by the analysis of the term by term convergence, as in [1]. Equation (2.16) follows by elementary but lengthy algebraic manipulations.

\section{Remarks}

1. The limit $f_{t}$ is a unique solution of the Boltzmann hierarchy in the sense that (2.18) makes sense. Equation (2.18) implies that $f_{t}$ solves

$$
f_{t}=S(t) f+\int_{0}^{t} S(t-s) C f_{s}
$$

In the factorization case, $f_{1, t}$ is then a mild solution of the Boltzmann equation (see, e.g., [2]).

2. With the techniques of this paper, the series (2.18) can be shown to converge also in the three dimensional case. The difficulty in proving the Boltzmann-Grad limit is related to an estimate of $\Omega^{d}(t)$ (see Proposition 3.1 below) which diverges in time in 3 dimensions. We believe that different topologies are needed to handle this problem.

3. As pointed out by Spohn [4], solutions of the Boltzmann hierarchy can be interpreted as statistical solutions of the Boltzmann equation, i.e. they describe the statistical evolution of individual solutions of the equation.

4. In our approach, as in [2], it is essential to work in all $\mathbb{R}^{2}\left(\right.$ or $\left.\mathbb{R}^{3}\right)$ in order to use the contractive character of free flow on a special class of functions. Compact domains require other ideas.

5. The result is not as strong as it may appear at first sight. Although time does not appear on the right-hand side of (2.26), the convergence is uniform only for $t<\tau$, because we have assumed $d \leqq 1 / \sqrt{2} \tau^{2}$.

6. As pointed out in [1], the gap between the topologies at time zero (2.13) and at time $t,(2.15)$ cannot be completely removed because of the irreversibility of the limit equation (see [5] for a discussion).

\section{Technical Details}

In this section we prove Proposition 2.1. We need two other propositions to do so. 
Proposition 3.1. For $d \leqq 1 / \sqrt{2} t^{2}$, the following inequalities hold:

$$
\begin{aligned}
& \left\|S^{d}(t) S(-t) f\right\|_{\beta, j} \leqq e^{2 \sqrt{ } 2 \beta}\|f\|_{\beta(1+d), j}, \\
& \left\|S(t) S^{d}(-t) f\right\|_{\beta, j} \leqq\|f\|_{\beta, j}
\end{aligned}
$$

Proof. First we establish two inequalities. Using the shorthand notation

$$
I(X):=\frac{1}{2} \Sigma x_{i}^{2}, \quad T(X):=\frac{1}{2} \Sigma v_{i}^{2},
$$

we have

$$
\begin{aligned}
& I\left(\phi_{t}^{d} X\right)+T\left(\phi_{t}^{d} X\right) \geqq I\left(\phi_{t} X\right)+T\left(\phi_{t} X\right), \\
& I\left(\phi_{t}^{d} X\right)+T\left(\phi_{t}^{d} X\right) \leqq I\left(\phi_{t} X\right)+\left(1+\sqrt{2} t^{2} d^{2}\right) T\left(\phi_{t} X\right)+\sqrt{2} .
\end{aligned}
$$

To verify (3.3) and (3.4), choose a phase point $X$ arbitrary but fixed. Then, for $t>0$, denote by $\left\{t_{i}\right\}_{i=1}^{k}, 0 \leqq t_{1}<t_{2} \cdots<t_{k} \leqq t$ the instants at which binary collisions take place (we exclude measure zero situations leading to multiple or simultaneous binary collisions). Let $y_{1}, y_{1}^{\prime} \cdots y_{k}, y_{k}^{\prime}, u_{1}, u_{1}^{\prime} \cdots u_{k}, u_{k}^{\prime}$ and $p_{1}, p_{1}^{\prime} \cdots p_{k}, p_{k}^{\prime}$ be the positions and the incoming and outgoing velocities respectively of the pairs of particles colliding at the times $t_{1}, \ldots, t_{k}$. Finally, we use the notations $\left\{x_{i}^{d}(t), v_{i}^{d}(t)\right\}_{i=1}^{j}$, $\left\{x_{i}(t), v_{i}(t)\right\}_{i=1}^{j}$ for the positions and velocities of $\phi_{t}^{d} X$ and $\phi_{t} X$ respectively, and put

$$
\left(\phi_{t_{i}}^{d} X\right)^{-}:=\lim _{t>t_{i}} \phi_{t}^{d} X, \quad\left(\phi_{t_{i}}^{d} X\right)^{+}:=\lim _{t>t_{i}} \phi_{t}^{d} X, \quad i=1, \ldots, k .
$$

Then, by energy conservation

$$
I\left(\phi_{t}^{d} X\right)=I\left(\left(\phi_{t_{k}}^{d} X\right)^{+}\right)+\left(t-t_{k}\right) \sum_{i=1}^{j} x_{i}^{d}\left(t_{k}\right) \cdot v_{i}^{d}\left(t_{k}\right)^{+}+\left(t-t_{k}\right)^{2} T(X) .
$$

Conservation of momentum implies

$$
\begin{aligned}
y_{k} p_{k}+y_{k}^{\prime} p_{k}^{\prime} & =y_{k}\left(p_{k}+p_{k}^{\prime}\right)+\left(y_{k}^{\prime}-y_{k}\right) p_{k}^{\prime}=y_{k}\left(u_{k}+u_{k}^{\prime}\right)+\left(y_{k}^{\prime}-y_{k}\right) p_{k}^{\prime} \\
& =y_{k} u_{k}+y_{k}^{\prime} u_{k}^{\prime}+\left(y_{k}^{\prime}-y_{k}\right)\left(p_{k}^{\prime}-u_{k}^{\prime}\right),
\end{aligned}
$$

therefore

$$
\begin{aligned}
I\left(\phi_{t}^{d} X\right)= & I\left(\left(\phi_{t_{k}}^{d} X\right)^{-}\right)+\left(t-t_{k}\right) \sum_{i=1}^{j} x_{i}^{d}\left(t_{k}\right) v_{i}^{d}\left(t_{k}\right)^{-} \\
& +\left(t-t_{k}\right)^{2} T(X)+\left(t-t_{k}\right)\left(y_{k}^{\prime}-y_{k}\right)\left(p_{k}^{\prime}-u_{k}^{\prime}\right) \\
= & I\left(\phi_{t-t_{k-1}}\left(\phi_{t_{k-1}}^{d} X\right)^{-}\right)+\left(t-t_{k}\right)\left(y_{k}^{\prime}-y_{k}\right)\left(p_{k}^{\prime}-u_{k}^{\prime}\right) .
\end{aligned}
$$

By iterating this procedure of elimination of collisions we obtain

$$
I\left(\phi_{t}^{d} X\right)=I\left(\phi_{t} X\right)+\sum_{i=1}^{k}\left(t_{i+1}-t_{i}\right)\left(y_{i}^{\prime}-y_{i}\right)\left(p_{i}^{\prime}-u_{i}^{\prime}\right)
$$

where $t_{k+1}:=t$. Because the last term on the right of (3.8) is positive, we obtain (3.3). On the other hand, the same term is estimated by

$$
2 \sqrt{2} \sum_{i=1}^{k}\left(t_{i+1}-t_{i}\right) d \sqrt{T(X)} \leqq \sqrt{2}\left(1+t^{2} T(X) d^{2}\right)
$$


and this proves (3.4). The statement of the Proposition now follows from the following chain of estimates, where we use that $\sqrt{2} t^{2} d<1$ :

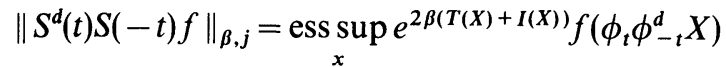

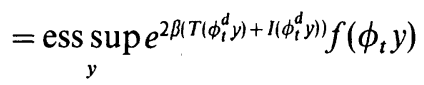

$$
\begin{aligned}
& \leqq \operatorname{ess} \sup e^{\left.2 \beta\left(I\left(\phi_{t}\right)\right)+(1+d) T\left(\phi_{t}\right)\right)} f\left(\phi_{t} y\right) e^{2 \sqrt{2 \beta}} \\
& \leqq e^{2 \sqrt{ } 2 \beta}\|f\|_{\beta(1+d), j} \text {. } \\
& \left\|S(t) S^{d}(-t) f\right\|_{\beta, j}=\underset{y}{\operatorname{ess} \sup } e^{2 \beta\left(T\left(\phi_{t} y\right)+I\left(\phi_{t} y\right)\right)} f\left(\phi_{t}^{d} y\right) \\
& \leqq \operatorname{ess} \sup e^{2 \beta\left(T\left(\phi_{t}^{d} y\right)+l\left(\phi_{t}^{d} y\right)\right)} f\left(\phi_{t}^{d} y\right) \\
& =\|f\|_{\beta, j} .
\end{aligned}
$$

Proposition 3.2. For $1>d \geqq 0, \beta>\beta^{\prime}>0, z^{\prime}>z>0$, there exists a positive constant $C_{0}$, not depending on $\beta, \beta^{\prime}, z, z^{\prime}, d$ and $t$ such that

$$
\begin{aligned}
\left\|V^{d}(t) f\right\|_{\beta^{\prime}}^{z^{\prime}} \leqq & C_{0} \lambda^{-1} z l(\beta)\left(\frac{1}{1+t^{3}}\right) \\
& \cdot\left\{\frac{1}{\left|\ln \frac{z}{z^{\prime}}\right|}+\frac{1}{\sqrt{\left(\beta-\beta^{\prime}\right)\left|\ln \frac{z}{z^{\prime}}\right|}}\right\}\|f\|_{\beta}^{z}, \text { for almost all } t,
\end{aligned}
$$

where $l(\beta)$ is given by (2.25).

Proof. By definition

$$
\begin{aligned}
& \left(V_{j, j+1}^{d}(t) f_{j+1}\right)\left(x_{1} \cdots x_{j}, v_{1} \cdots v_{j}\right) \\
& =(N-j) d \sum_{k=1}^{j} \int d n \int d v_{j+1} n \cdot\left(v_{k}-v_{j+1}\right) f_{j+1} \\
& \quad \cdot\left(x_{1} \cdots x_{j}, x_{k}+n d+\left(v_{k}-v_{j+1}\right) t, v_{1}, \ldots, v_{j+1}\right) .
\end{aligned}
$$

Then

$$
\begin{aligned}
& \exp \left(\beta^{\prime}\left(\sum_{i=1}^{j} v_{i}^{2}+\sum_{i=1}^{j} x_{i}^{2}\right)\right)\left|\left(V_{j, j+1}^{d}(t) f_{j+1}\right)\left(x_{1} \cdots x_{j}, v_{1} \cdots v_{j}\right)\right| \\
& \leqq \lambda^{-1} \sum_{k=1}^{j} \int d n \int d v_{j+1}\left|v_{k}-v_{j+1}\right|\left\|f_{j+1}\right\|_{\beta, j+1} \cdot \\
& \quad \cdot \exp \left[-\left(\beta-\beta^{\prime}\right) \sum_{i=1}^{j}\left(x_{i}^{2}+v_{i}^{2}\right)\right] \exp \left[-\beta v_{j+1}^{2}\right] \\
& \quad \cdot \exp \left[-\beta\left(x_{k}+n d+\left(v_{k}-v_{j+1}\right) t\right)^{2}\right] .
\end{aligned}
$$


For $t \leqq 1$, we have

$$
\begin{aligned}
& \int d v_{j+1}\left|v_{k}-v_{j+1}\right| \exp \left[-\beta\left(x_{k}+n d+\left(v_{k}-v_{j+1}\right) t\right)^{2}\right] \exp \left[-\beta v_{j+1}^{2}\right] \\
& \quad \leqq \int d v_{j+1}\left(\left|v_{k}\right|+\left|v_{j+1}\right|\right) e^{-\beta v_{j+1}^{2}} \\
& \quad \leqq \pi \beta^{-1}\left|v_{k}\right|+C_{1} \beta^{-3 / 2} \\
& \quad \leqq C(\beta)\left(1+\left|v_{k}\right|\right) \cdot \frac{1}{1+i^{3}}
\end{aligned}
$$

with

$$
C_{1}=\int_{\mathbb{R}^{2}}|x| e^{-x^{2}} d x, \quad C(\beta)=2 \max \left(C_{1} \beta^{-3 / 2}, \pi \beta^{-1}\right) .
$$

For $t>1$, the same integral is bounded by

$$
\begin{gathered}
\int d v_{j+1}\left|v_{k}-v_{j+1}\right| e^{-\beta\left(x_{k}+n d+\left(v_{k}-v_{j+1}\right) t\right)^{2}} \leqq t^{-3} \int d \xi\left\{|\xi|+\left|x_{k}+n d\right|\right\} e^{-\beta \xi^{2}} \\
\leqq t^{-3}\left(C_{1} \beta^{-3 / 2}+\left(\left|x_{k}\right|+d\right) \beta^{-1}\right) \leqq 2 \cdot C(\beta)\left(2+\left|x_{k}\right|\right) \frac{1}{1+t^{3}}
\end{gathered}
$$

(here we have used that $d<1$ ).

Inserting (3.15) and (3.16) into (3.14) we obtain

$$
\begin{aligned}
& \left\|V_{j, j+1}^{d}(t) f_{j+1}\right\|_{\beta^{\prime}, j} \leqq C(\beta) \lambda^{-1} 2 \pi\left(\frac{1}{1+t^{3}}\right) \\
& \quad \sup _{\substack{x_{1} \ldots x_{j} \\
v_{1} \ldots v_{j}}} \sum_{k=1}^{j}\left\{3+\left|x_{k}\right|+\left|v_{k}\right|\right\} \cdot e^{-\left(\beta-\beta^{\prime}\right) \sum_{i=1}^{j}\left(x_{i}^{2}+v_{i}^{2}\right)}\left\|f_{j+1}\right\|_{\beta, j+1} .
\end{aligned}
$$

There is a constant $C_{2}>0$ such that

$$
\sum_{i=1}^{j}\left|x_{i}\right| e^{-\gamma \sum_{i=1}^{j} x_{i}^{2}} \leqq C_{2} \sqrt{\frac{j}{\gamma}}
$$

and it follows that

$$
\left\|V_{j, j+1}^{d}(t) f_{j+1}\right\|_{\beta^{\prime}, j} \leqq C_{3} \cdot C(\beta) \lambda^{-1}\left(\frac{1}{1+t^{3}}\right)\left(j+\sqrt{\frac{j}{\beta-\beta^{\prime}}}\right)\left\|f_{j+1}\right\|_{\beta, j+1}
$$

with some $C_{3}>0$. Therefore

$$
\left\|V^{d}(t) f\right\|_{\beta^{\prime}}^{z^{\prime}} \leqq C_{3} C(\beta)\|f\|_{\beta^{z}}^{z} z \lambda^{-1}\left(\frac{1}{1+t^{3}}\right) \cdot\left[\sup _{j}\left(j+\sqrt{\frac{j}{\beta-\beta^{\prime}}}\right)\left(\frac{z}{z^{\prime}}\right)^{j}\right] .
$$

It is a simple exercise to verify that

$$
\begin{aligned}
\sup _{j}\left(j+\sqrt{\frac{j}{\beta-\beta^{\prime}}}\right)\left(\frac{z}{z^{\prime}}\right)^{j} & \leqq \sup _{j} j\left(\frac{z}{z^{\prime}}\right)^{j}+\sup _{j} \sqrt{\frac{j}{\beta-\beta^{\prime}}}\left(\frac{z}{z^{\prime}}\right)^{j} \\
& \leqq \frac{1}{\left|\ln \frac{z^{\prime}}{z}\right|}+\frac{1}{\sqrt{\left|\ln \frac{z^{\prime}}{z}\right|\left(\beta-\beta^{\prime}\right)}}
\end{aligned}
$$


and this completes the proof of Proposition 3.2.

Proposition 2.1 follows trivially from Propositions 3.1 and 3.2.

\section{Appendix}

In this appendix we sketch a derivation of Eqs. (2.4) for hard sphere particle systems. A more detailed version of this derivation will be published elsewhere. A more intricate derivation was given in [6].

Our starting point is the flow $\phi_{t}^{N}$ describing the dynamics of $N$ spheres of diameter $d>0$ in $\mathbb{R}^{v}(v=2,3)$. We denote by

$$
\Gamma_{N}=\left\{X\left|X=\left(x_{i}, v_{i}\right)_{i=1}^{N}, x_{i}, v_{i} \in \mathbb{R}^{v},\right| x_{i}-x_{j} \mid \geqq d \forall i \neq j\right\}
$$

the phase space of the system. It has been proved in ref. [7] that the set of all orbits $\phi_{t}^{N}(X), X \in \Gamma_{N}, t \in \mathbb{R}$, which lead to either grazing or multiple collisions, has measure 0 in $\Gamma_{N}$. We denote the set of all other (the "good") phase points by $\check{\Gamma}_{N}$.

For fixed $k<N$, let

$$
\begin{aligned}
& \mathscr{F}_{i j}^{+(-)}=\left\{X \in \Gamma_{N}|| x_{i}-x_{j} \mid=d,\left(x_{i}-x_{j}\right) \cdot\left(v_{i}-v_{j}\right) \leqq 0(>0)\right\} \\
& \text { for some } i \in\{1, \ldots, k\}, j \in\{k+1, \ldots, N\}
\end{aligned}
$$

be the set of all points in $\check{\Gamma}_{N}$ which display an outgoing (ingoing) collision between the $i$-particle and the $j$-particle. Furthermore, we put

$$
\mathscr{F}^{+(-)}=\bigcup_{i=1}^{k} \bigcup_{j=k+1}^{N} \mathscr{F}_{i j}^{+(-)}, \quad \text { and } \quad \mathscr{F}^{+}=\mathscr{F}^{+} \cup \mathscr{F}^{-} \text {. }
$$

We fix the origin of the time axis and split $\stackrel{\circ}{\Gamma}_{N}$ into two disjoint sets:

$$
\check{\Gamma}_{N}=\Gamma^{\infty} \cup \tilde{\Gamma},
$$

where

$$
\Gamma^{\infty}=\left\{X=\left(X^{k}, X^{N-k}\right) \mid X^{k} \in \Gamma_{k}, X^{N-k} \in \Gamma_{N-k}, \phi_{-t}^{N} X=\left(\phi_{-t}^{k} X^{k}, \phi_{-t}^{N-k} X^{N-k}\right), t \geqq 0\right\}
$$

is the set of all phase points for which the particles from the group $1, \ldots, k$ never interacted in the past with the rest of the particles, and

$$
\tilde{\Gamma}=\Gamma_{N} \backslash \Gamma^{\infty} \text {. }
$$

For $X \in \tilde{\Gamma}$, the following representation (known as special flow representation) is natural. Let

$$
s=s(X)=\min \left\{t \mid \phi_{-t}^{N}(X)=Y \in \mathscr{F}^{+}\right\}, \quad \varphi(Y)=\min \left\{t \mid \phi_{t}^{N}(Y) \in \mathscr{F}^{-}, Y \in \mathscr{F}^{+}\right\} .
$$

The mapping $\psi: \tilde{\Gamma} \rightarrow\left\{(Y, s) \mid Y \in \mathscr{F}^{+}, 0 \leqq s \leqq \varphi(Y)\right\}, X \rightarrow(Y, s)$, is then one to one and bimeasurable. For $g \in L^{1}(\widetilde{\Gamma})$, we have

$$
\int_{\tilde{\Gamma}} g(X) d X=\int_{\mathscr{F}^{+}} d \sigma^{+}(y) \int_{0}^{\varphi(Y)} d s g\left(\psi^{-1}(Y, s)\right),
$$


where $d \sigma^{+}$denotes the following measure on $\mathscr{F}^{+}$:

$$
d \sigma^{+}=d X^{k} \sum_{j=k+1}^{N} d x_{k+1} \cdots d x_{j-1} d x_{j+1} \cdots d x_{N} d v_{k+1} \cdots d v_{N} d y_{j} \cdot n_{i j} \cdot\left(v_{j}-v_{i}\right) .
$$

Here, $d X^{k}$ is the Lebesgue measure on $\Gamma_{k}$, and $d y_{j}$ stands for the Lebesgue measure on the sphere with radius $d$ and center $x_{i}$, and $n_{i j}=\left(x_{j}-x_{i}\right) /\left|x_{j}-x_{i}\right|$. The right-hand side of (A.8) defines also a (negative) measure on $\mathscr{F}^{-}$, denoted by $d \sigma^{-}$. For the proof of the above statements, see e.g. [8].

The advantage of the special flow representation consists of the fact that

$$
\phi_{t}^{N}(X) \stackrel{\psi}{\rightarrow}(Y, s+t) \quad \text { if } \quad X \stackrel{\psi}{\rightarrow}(Y, s) \quad \text { and } \quad s+t<\varphi(Y) .
$$

Moreover, there exists a map $T: \mathscr{F}^{+} \rightarrow \mathscr{F}^{+}$(the "displacement function" for the Poincaré mapping associated with the section $\mathscr{F}^{+}$), which is $d \sigma^{+}$-preserving and for which

$$
\phi_{t}^{N}(X) \stackrel{\psi}{\rightarrow}(T Y, \tau)
$$

if $\varphi(Y) \leqq s+t \leqq \varphi(T Y)$ and $\tau=s+t-\varphi(Y)$. The flow $\phi_{t}^{N}$, restricted to $\tilde{\Gamma}$, gives rise to a strongly continuous group of unitary operators whose generator we denote by $\tilde{\mathscr{L}}$. We denote its image on $L^{2}\left(\psi(\tilde{\Gamma}), d \sigma^{+} d t\right)$ by $D$. For a sufficiently smooth function $v=v(Y, s)$, we have

$$
(D v)(Y, s)=\frac{\partial}{\partial s} v(Y, s)
$$

The domain $\mathscr{D}(D)$ can be completely characterized:

Lemma A. $\mathscr{D}(D)$ consists of all functions of the type

$$
v(Y, t)=v_{0}(Y)+\int_{0}^{t} d \operatorname{sh}(Y, s)
$$

where

$$
\begin{aligned}
& v_{0} \cdot \sqrt{\varphi} \in L^{2}\left(\mathscr{F}^{+}, d \sigma^{+}\right), \\
& v_{0}(Y)+\int_{0}^{\varphi(Y)} h(Y, s) d s=v_{0}(T Y)
\end{aligned}
$$

and

$$
h=D v \in L^{2}\left(\psi(\tilde{\Gamma}), d \sigma^{+} d t\right) .
$$

The proof of Lemma A will be given at the end of the appendix.

We now consider a probability density $\mu_{0} \in L^{2}\left(\Gamma_{N}\right)$ which is differentiable along the trajectories, i.e. with $\mu_{t}(X)=\mu_{0}\left(\phi_{-t}^{N} X\right)$, we have

$$
\left.\frac{d}{d t} \mu_{t}(X)\right|_{t=0}=:\left(\mathscr{L}_{N} \mu_{0}\right)(X) \in L^{2}\left(\Gamma_{N}\right)
$$

Then the restriction of $\mu_{0}$ to $\tilde{\Gamma}$ belongs to $\mathscr{D}(\tilde{\mathscr{L}})$. For a bounded function $u_{k} \in \mathscr{D}\left(\mathscr{L}_{k}\right)$ 
which depends only on the first $k$ particles, we have

$$
\begin{aligned}
\frac{d}{d t} \int_{T^{\prime}} u_{k} \mu_{t} d X= & \frac{d}{d t} \int_{\mathscr{F}^{+}} d \sigma^{+}(Y) \int_{0}^{\varphi(Y)} d s u_{k}\left(\psi^{-1}(Y, s)\right) \mu_{t}\left(\psi^{-1}(Y, s)\right) \\
= & -\int_{\mathscr{F}^{+}} d \sigma^{+}(Y) \int_{0}^{\varphi(Y)} d s\left(\mathscr{L}_{k} u_{k}\right)\left(\psi^{-1}(Y, s)\right) \mu_{t}\left(\psi^{-1}(Y, s)\right) \\
& +\int_{\mathscr{F}^{+}} d \sigma^{+}(Y)\left\{u _ { k } \left(\psi ^ { - 1 } ( Y , \varphi ( Y ) ) \mu _ { t } \left(\psi^{-1}(Y, \varphi(Y))\right.\right.\right. \\
& \left.-u_{k}\left(\psi^{-1}(Y, 0)\right) \mu_{t}\left(\psi^{-1}(Y, 0)\right)\right\},
\end{aligned}
$$

since $\left.u_{k} \mu_{t}\right|_{\Gamma} \in \mathscr{D}(\tilde{\mathscr{L}})$ and the integration by parts is possible by Lemma A.

With $F=u_{k} \cdot \mu_{t}$, we have by Liouville's theorem,

$$
\int_{\mathscr{F}^{+}} d \sigma^{+}(Y) F(Y, \varphi(Y))=-\int_{\mathscr{F}^{-}} d \sigma^{-}(Y) F(Y, 0),
$$

and so the last integral in the right-hand side of (A.15) reduces to

$$
\begin{gathered}
\int d X^{k} u\left(X^{k}\right) \sum_{j=k+1}^{N} d x_{k+1} \cdots d x_{j-1} d x_{j+1} \cdots d x_{N} d y_{j} n_{i j}\left(v_{j}-v_{i}\right) \\
\cdot d v_{k+1} \cdots d v_{N} \mu_{t}^{N}\left(x_{1} \cdots x_{j-1} y_{j} x_{j+1} \cdots x_{N}, v_{1} \cdots v_{N}\right) .
\end{gathered}
$$

We notice that by the continuity property of $\mu_{0}$ along the trajectories, $\mu_{t}$ is $d \sigma^{+}$a.e. defined in $\mathscr{F}^{+}$. Therefore,

$$
\begin{aligned}
& f_{t}^{k+1}\left(x_{1}, \ldots, x_{k}, x_{k}+n d, v_{1}, \ldots, v_{k+1}\right) \\
& \quad=\int d X^{N-k+1} \mu_{t}\left(x_{1} \cdots x_{k}, x_{k}+n d, v_{1} \cdots v_{k+1} ; X^{N-k+1}\right)
\end{aligned}
$$

is a.e. defined. (Eqn. A.17) can be written in the form

$$
\int d X^{k} u_{k}\left(X^{k}\right) C_{k, k+1} f_{t}^{k+1}\left(X^{k}\right) .
$$

Finally

$$
\begin{aligned}
& \frac{d}{d t} \int d X^{k} u_{k}\left(X^{k}\right) f_{t}^{k}\left(X^{k}\right)=\frac{d}{d t} \int_{\Gamma^{\infty}} d X u_{k}(X) \mu_{t}(X)+\frac{d}{d t} \int_{\Gamma} d X u_{k}(X) \mu_{t}(X) \\
& \quad=-\int_{\Gamma^{\infty}} d X\left(\mathscr{L}_{k} u_{k}\right)(X) \mu_{t}(X)+\text { right-hand side of }(\mathrm{A} .15) \\
& \quad=-\int d X^{k}\left(\mathscr{L}_{k} u_{k}\right)\left(X^{k}\right) f_{t}^{k}\left(X^{k}\right)+\int d X^{k} u_{k}\left(X^{k}\right) C_{k, k+1} f_{t}^{k+1}\left(X^{k}\right)
\end{aligned}
$$

where $\mathscr{L}_{k}$ is the generator associated with the flow $\phi_{t}^{k}$. The series expansion $(2.4)_{2}$ satisfies the same differential equation for all sufficiently smooth $u_{k}$. Thus the joint distributions $f_{t}^{k}$ defined by $\mu_{t}$ must coincide a.e. with the corresponding expressions given by the series expansion.

Proof of Lemma A. It is easy to verify that $D$ restricted to the functions of type (A.12) and satisfying (A.13) (let us denote this set by $\mathscr{D}_{0}$ ) is antisymmetric. Moreover, this domain is invariant for the flow $\phi_{t}^{N}$. It remains to show that if $w \in \mathscr{D}\left(D^{*}\right)$, then $w \in \mathscr{D}_{0}$. 
For all $v \in \mathscr{D}_{0}$,

$$
\begin{aligned}
\int_{\mathscr{F}^{+}} d \sigma^{+} & \int_{0}^{\varphi(Y)} d t w(Y, t)(D v)(Y, t)=-\int_{\mathscr{F}^{+}} d \sigma^{+} \int_{0}^{\varphi(Y)} d t\left(D^{*} w\right)(Y, t) v(Y, t) \\
=- & \int_{\mathscr{F}^{+}} d \sigma^{+} \int_{0}^{\varphi(Y)} d t\left(D^{*} w\right)(Y, t) v(Y, 0)-\int_{\mathscr{F}^{+}} d \sigma^{+} \int_{0}^{\varphi(Y)} d t\left(D^{*} w\right)(Y, t) \int_{0}^{t} d s(D v)(Y, s) \\
=- & \int_{\mathscr{F}^{+}} d \sigma^{+} \int_{0}^{\varphi(Y)} d t\left(D^{*} w\right)(Y, t) v(Y, 0)-\int_{\mathscr{F}^{+}} d \sigma^{+} \int_{0}^{\varphi(Y)} d s(D v)(Y, s) \int_{s}^{\varphi(Y)} d t\left(D^{*} w\right)(Y, t) .
\end{aligned}
$$

The above identity is valid for all $v$ such that $v(Y, 0)=0$. Therefore $w(Y, t)+\int_{t}^{\varphi(Y)} d s$ $\left(D^{*} w\right)(Y, s) \in L^{2}\left(\psi(\tilde{\Gamma}), d \sigma^{+} d t\right)$ is only dependent on $Y$, i.e. there is a function $w_{0} \in L^{2}\left(\mathscr{F}^{+}, d \sigma^{+} / \varphi\right)$ such that

$$
w(Y, t)=w_{0}(Y, \varphi(Y))-\int_{t}^{\varphi(Y)} d s\left(D^{*} w\right)(Y, s)
$$

Inserting this expression in (A.21), we are led to the identity

$$
\begin{aligned}
\int_{\mathscr{F}^{+}} d \sigma^{+}(Y) w(Y, \varphi(Y))[v(Y, \varphi(Y))-v(Y, 0)] \\
=\int_{\mathscr{F}^{+}} d \sigma^{+}(Y) v(Y, 0)[w(Y, 0)-w(Y, \varphi(Y))]
\end{aligned}
$$

which is equivalent to

$$
\int_{\mathscr{F}^{+}} d \sigma^{+}(Y)\left[w\left(T^{-1} Y, \varphi\left(T^{-1} Y\right)\right)-w(Y, 0)\right] v(Y, 0)=0 .
$$

Since $v(\cdot, 0)$ is arbitrary in $L^{2}\left(\mathscr{F}^{+}, d \sigma^{+} / \varphi\right)$, the proof is complete.

Acknowledgements. We would like to express our gratitude to Carlo Cercignani, who first suggested this problem, to Enrico Presutti and Marvin Shinbrot for fruitful discussions, and to Lucio Micheli and Brunello Tirozzi, who contributed greatly to the proof of the BBGKY-hierarchy given in the appendix. One of the authors (R.I.) wants to acknowledge the generosity of the Italian C.N.R. and the hospitality at the University of Rome, where part of this work was done.

\section{References}

1. Lanford, O. III: Time evolution of large classical systems. Moser, E. J. (ed.) Lecture Notes in Physics, Vol. 38, pp. 1-111. Berlin, Heidelberg, New York: Springer 1975

2. Illner, R., Shinbrot, M.: The Boltzmann equation: Global existence for a rare gas in an infinite vacuum. Commun. Math. Phys. 95, 217-226 (1984)

3. Cercignani, C.: On the Boltzmann equation for Rigid spheres. Transp. Theory Stat. Phys. 2, 211-225 (1972)

4. Spohn, H.: Boltzmann hierarchy and Boltzmann equation. In: Kinetic theories and the Boltzmann equation. Lecture Notes in Mathematics, Vol. 1048, Montecatini 1981 (1984)

5. Illner, R., Neunzert, H.: The concept of irreversibility in the kinetic theory of gases, preprint 1985 
6. Spohn, H.: On the integrated form of the RBGKY hierarchy for hard spheres, unpublished

7. Alexander, R. K.: The infinite hard sphere system, Ph.D. Thesis, Department of Mathematics. Berkeley: University of California, 1975

8. Marchioro, C., Pellegrinotto, A., Presutti, E., Pulvirenti, M.: On the dynamics of particles in a bounded region: A measure theoretical approach. J. Math. Phys. 17, 647-652 (1976)

Communicated by J. L. Lebowitz

Received May 31, 1985 
